\title{
Relação entre aptidão cardiorrespiratória e indicadores de adiposidade corporal em adolescentes
}

\author{
Relationship between cardiorespiratory fitness and indicators of body adiposity in adolescents
}

\author{
Enio Ricardo V. Ronque ${ }^{1}$, Edilson Serpeloni Cyrino ${ }^{1}$, Arnaldo Luis Mortatti ${ }^{2}$, Alexandre Moreira ${ }^{3}$, Ademar Avelar ${ }^{4}$, \\ Ferdinando Oliveira Carvalho ${ }^{5}$, Miguel de Arruda ${ }^{6}$
}

\section{RESUMO}

Objetivo: Analisar a relação entre aptidão cardiorrespiratória e indicadores de adiposidade corporal em adolescentes de ambos os sexos.

Métodos: 78 sujeitos ( 45 rapazes e 33 moças) foram avaliados quanto à massa corporal, estatura e dobras cutâneas tricipital e subescapular. O índice de massa corpórea e a somatória das duas dobras cutâneas foram calculados. A aptidão cardiorrespiratória foi analisada pelo teste de vai-e-vem de 20 metros de vários estágios e a estimativa do consumo máximo de oxigênio foi analisada por uma equação específica. Para a comparação entre os grupos, foi empregado o teste de Mann-Whitney e, para verificar a relação entre a aptidão cardiorrespiratória e os indicadores de adiposidade corporal, utilizou-se o coeficiente de correlação de Spearman.

Resultados: Os adolescentes com alta aptidão cardiorrespiratória apresentaram sistematicamente valores mais baixos de gordura corporal em relação a seus pares com baixa aptidão cardiorrespiratória $(p<0,05)$, com diferenças variando entre 36 e $42 \%$ para os rapazes e entre 14 e $33 \%$ para as moças. Correlações negativas, moderadas e estatisticamente significativas foram verificadas entre os indicadores de aptidão cardiorrespiratória e de adiposidade corporal para ambos os sexos, com exceção do índice de massa corpórea.

Conclusões: Os resultados sugerem que o aumento da adiposidade corporal pode influenciar negativamente a aptidão cardiorrespiratória em adolescentes de ambos os sexos.

Instituição: Universidade Estadual de Londrina (UEL), Londrina, PR, Brasil 'Doutor; Professor de Pós-Graduação em Educação Física da UEL, Londrina, PR, Brasil

2Doutorando em Saúde da Criança e do Adolescente na Universidade Estadual de Campinas (Unicamp), Campinas, SP, Brasil

${ }^{3}$ Doutor; Professor de Pós-Graduação em Educação Física da Universidade de São Paulo (USP), São Paulo, SP, Brasil

${ }^{4}$ Mestre; Professor de Educação Física na UEL, Londrina, PR, Brasil

${ }^{5}$ Doutorando em Educação Física na Universidade Católica de Brasília (UCB), Brasília, DF, Brasil

${ }^{6}$ Doutor; Professor de Pós-Graduação em Educação Física da Unicamp, Campinas, SP, Brasil
Palavras-chave: aptidão física; adiposidade; saúde do adolescente.

\section{ABSTRACT}

Objective: To analyze the relationship between cardiorespiratory fitness and indicators of body adiposity in adolescents of both genders.

Methods: 78 subjects ( 45 boys and 33 girls) were evaluated for body mass, height and triceps and subscapular skinfold thickness. Body mass index and the sum of the two skinfolds thickness were determined. The cardiorespiratory fitness was determined by the maximal multistage 20 meters shuttle run test, and the estimate of maximal oxygen uptake was evaluated by a specific equation. Groups were compared by Mann-Whitney test. The Spearman correlation index was applied to verify the relationship between cardiorespiratory fitness and the indicators of body adiposity.

Results: The adolescents with high cardiorespiratory fitness presented lower values of body fat than their peers with low cardiorespiratory fitness $(p<0.05)$, with differences varying between 36 to $42 \%$ for boys and 14 to $33 \%$ for girls. Statistically significant negative and moderate correlations were noted between the indicators of cardiorespiratory fitness and the body adiposity for both genders, with the exception of body mass index.

Conclusions: These results suggest that the body adiposity may have a negative influence on the cardiorespiratory fitness of adolescents of both genders.

Endereço para correspondência:

Enio Ricardo V. Ronque

Rodovia Celso Garcia Cid, km 380, Campus Universitário

CEP 86051-990 - Londrina/PR

E-mail: enioronque@uel.br

Conflito de interesse: nada a declarar

Recebido em:30/6/2009

Aprovado em:30/11/2009 
Key-words: physical fitness; adiposity; adolescent health.

\section{Introdução}

Nos últimos anos, o avanço científico e tecnológico, bem como o aumento da insegurança em ruas e praças, principalmente nos grandes centros urbanos, têm contribuído para mudanças no estilo de vida de crianças e adolescentes. Assim, grande parte das horas de lazer e tempo livre dessa população é destinada a jogos, diversões e brincadeiras predominantemente passivos em detrimento da participação em programas de exercícios físicos, esportes organizados e não-organizados, o que tem resultado em importante redução dos níveis de atividade física habitual ${ }^{(1,2)}$.

Tal fato tem gerado grande preocupação entre profissionais e pesquisadores das áreas de saúde pública, uma vez que o estilo de vida tipicamente sedentário apresenta forte relação com o aumento da prevalência de sobrepeso/obesidade nessa população, favorecendo o desenvolvimento e/ou agravamento de inúmeras disfunções crônico-degenerativas, tais como hipertensão arterial, diabetes tipo II, hipercolesterolemia, hiperlipidemia e doenças cardiovasculares ${ }^{(3)}$. Além disso, o excesso de gordura corporal adquirido na infância e adolescência tende a permanecer ao longo da idade adulta ${ }^{(4)}$.

Adicionalmente, níveis relativamente reduzidos de atividade física habitual em jovens podem afetar de modo negativo diferentes parâmetros relacionados à saúde, entre os quais a aptidão cardiorrespiratória $(\mathrm{ACR})^{(5,6)}$. Vale destacar que, em adultos, uma boa ACR parece exercer um efeito protetor, sobretudo no que concerne ao desenvolvimento de disfunções metabólicas ${ }^{(7)}$, além de reduzir o risco de mortalidade por todas as causas tanto em homens quanto em mulheres ${ }^{(8)}$.

Em contrapartida, em crianças e adolescentes, alguns estudos demonstram que baixos índices de ACR podem se associar fortemente ao aumento dos fatores de risco cardiovascular e síndrome metabólica ${ }^{(9-12)}$. Acredita-se, também, que baixos índices da ACR na infância e na adolescência estejam associados ao aumento do risco cardiovascular na idade adulta ${ }^{(13)}$.

Nesse sentido, alguns pesquisadores advogam que a quantidade de gordura corporal pode exercer uma importante influência na relação entre ACR e fatores de risco cardiovascular, tanto em indivíduos jovens quanto em adultos ou, ainda, durante o período de transição entre essas fases da vida ${ }^{(14-16)}$. Embora alguns estudos realizados em outros países tenham investigado a relação entre ACR e gordura corporal $^{(17,18)}$, não existem informações consistentes a esse respeito particularmente em adolescentes brasileiros. Assim, o propósito deste estudo foi analisar possíveis relações entre ACR e indicadores de adiposidade corporal em adolescentes brasileiros de ambos os sexos.

\section{Método}

Trata-se de um estudo transversal, realizado de setembro a novembro de 2002, utilizando o banco de dados de uma pesquisa de delineamento longitudinal misto (multiple longitudinal design) intitulado "Análise do crescimento e da aptidão física relacionada à saúde em escolares de alto nível socioeconômico", realizado em Londrina (PR), Brasil, entre 2002 e 2006.

Para a realização desse projeto, foi selecionada uma escola da região central do município que atendia aos critérios estabelecidos para o desenvolvimento de um estudo com características longitudinais e apresentava infraestrutura adequada para a coleta de dados.

Todos os adolescentes matriculados na referida escola, pertencentes à faixa etária de 15 anos, foram convidados a participar do estudo ( $n=109)$, independentemente da classificação socioeconômica. Os critérios de inclusão dos sujeitos na coleta dos dados foram: demonstrar vontade em participar do estudo, estar matriculado e frequentar normalmente as atividades da referida instituição, além de apresentar a autorização dos pais ou responsáveis. Foram excluídos das análises os sujeitos que não pertenciam à faixa etária de interesse $(\mathrm{n}=12)$, tinham algum problema físico que os impedisse de se submeter à administração de testes motores $(n=2)$, estavam ausentes no dia da avaliação $(n=4)$ e não apresentaram a autorização dos pais ou responsáveis $(n=13)$.

Este estudo foi desenvolvido em conformidade com as instruções contidas na Resolução 196/96 do Conselho Nacional de Saúde para estudos com seres humanos, do Ministério da Saúde, tendo sido aprovado pelo Comitê de Ética em Pesquisa da Universidade Estadual de Campinas.

Assim, a amostra foi constituída por 78 adolescentes (45 rapazes e 33 moças), na faixa etária de 15 anos. A idade decimal em anos foi calculada conforme os procedimentos descritos por Ross e Marfell-Jones ${ }^{(19)}$, sendo o grupo etário estabelecido por meio de intervalos decimais 0,50 a 0,49 (ou seja, idade $\geq 14,50$ e $<15,50$ anos). O número de adolescentes avaliados foi suficiente para identificar correlação moderada, da ordem de $r=0,68^{(17)}$, com $\alpha=0,05$ e $\beta=0,20$ para cada sexo. O cálculo do tamanho da amostra foi realizado por meio do programa Bioestat 3.0. 
A massa corporal foi obtida em uma balança digital da marca Filizola, com precisão de $0,05 \mathrm{~kg}$, e a estatura foi determinada em um estadiômetro de madeira com precisão de $0,1 \mathrm{~cm}$, de acordo com procedimentos padronizados e descritos na literatura $^{(20)}$. A partir dessas informações, o índice de massa corpórea (IMC) foi calculado.

A espessura das dobras cutâneas tricipital (DCTR) e subescapular (DCSE) foram medidas para análise da adiposidade subcutânea. Tais medidas foram realizadas por um único avaliador experiente com um adipômetro científico da marca Cescorf, de fabricação nacional, conforme os procedimentos descritos por Harrison et al ${ }^{(21)}$. O coeficiente teste-reteste excedeu 0,95 para cada um dos pontos anatômicos, com erro de medida de no máximo $\pm 1,0 \mathrm{~mm}$. O somatório das dobras cutâneas ( $(D C)$ foi utilizado como indicador de adiposidade corporal, assim como a gordura corporal relativa (\%GORD), estimada a partir da equação proposta por Boileau et al ${ }^{(22)}$.

A ACR foi determinada de acordo com o desempenho no teste de corrida de vai-e-vem de 20 metros (SR-20m), conforme procedimentos descritos por Leger et al ${ }^{(23)}$, com a estimativa do consumo máximo de oxigênio $\left(\mathrm{VO}_{2} \max \right)$ determinada por equação específica ${ }^{(24)}$. Para a classificação da ACR, foram adotados os pontos de corte recomendados pelo Fitnessgram ${ }^{(25)}$, com base no número de voltas realizadas durante o teste (Tabela 1).

Para efeito de análise, os sujeitos foram classificados em baixa ACR (não atenderam ao critério estabelecido) e alta ACR (atenderam ao critério). Vale destacar que o teste SR$20 \mathrm{~m}$ tem demonstrado boa validade $[\mathrm{r}=0,71$ e $\mathrm{EPM}=5,9 \mathrm{~mL} /$ $\mathrm{kg} / \mathrm{min}]^{(24)}$ para estimar a ACR, bem como uma relação bastante aceitável entre $\mathrm{o} \mathrm{VO}_{2}$ medido diretamente com o número de voltas em adolescentes, tanto para os rapazes $(\mathrm{r}=0,63)$ quanto para as moças $(\mathrm{r}=0,58)^{(26)}$.

As informações encontradas foram expressas em valores de mediana e diferenças interquartis (Q3-Q1), visto que o teste de Shapiro-Wilk não confirmou a normalidade na distribuição dos dados. O teste do qui-quadrado foi adotado para comparar proporções de adolescentes que atenderam e que não atenderam aos critérios estabelecidos para ACR. Para analisar as variáveis numéricas dos grupos com alta e baixa ACR, foi empregado o teste de Mann-Whitney. Para verificar possíveis relações entre indicadores de ACR (SR$20 \mathrm{~m}$ e $\mathrm{VO}_{2}$ máx) e de adiposidade corporal, foi utilizado o coeficiente de correlação de Spearman. O nível de significância adotado foi de $5 \%$. As informações foram processadas no pacote computacional SPSS, versão 17.0.

\section{Resultados}

Dos 78 adolescentes investigados, $58 \%$ eram do sexo masculino (idade: $15,0 \pm 0,2$ anos; massa corporal: $62,3 \pm 9,8 \mathrm{~kg}$; estatura: $171,4 \pm 0,1 \mathrm{~cm}$; IMC: $\left.21,2 \pm 3,4 \mathrm{~kg} / \mathrm{m}^{2}\right)$ e $42 \%$ do sexo feminino (idade: $15,1 \pm 0,2$ anos; massa corporal: $52,3 \pm 8,5 \mathrm{~kg}$; estatura: $159,9 \pm 0,1 \mathrm{~cm}$; IMC: $20,4 \pm 3,2 \mathrm{~kg} / \mathrm{m}^{2}$ ).

As características físicas dos adolescentes, de acordo com o nível de ACR e sexo, estão apresentadas na Tabela 2. Verificou-se que $42 \%$ dos rapazes e $60 \%$ das moças atenderam aos critérios estabelecidos pelo Fitnessgram ${ }^{(25)}$ para ACR. O teste de qui-quadrado não indicou nenhuma diferença significativa entre os adolescentes classificados com alta e baixa

Tabela 1 - Pontos de corte com base no número mínimo de voltas recomendado pelo Fitnessgra* no teste SR-20m por sexo e idade

\begin{tabular}{lcccccc}
\hline & $\mathbf{1 0}$ anos & $\mathbf{1 1}$ anos & $\mathbf{1 2}$ anos & $\mathbf{1 3}$ anos & $\mathbf{1 4}$ anos & $\mathbf{1 5}$ anos \\
\hline Rapazes & 23 & 23 & 32 & 41 & 41 & 51 \\
Moças & 15 & 15 & 23 & 23 & 23 & 23 \\
\hline
\end{tabular}

*Adaptado do The prudential Fitnessgram test administration manual.

Tabela 2 - Características físicas dos adolescentes, segundo aptidão cardiorrespiratória (ACR) e gênero

\begin{tabular}{lcccccc}
\hline & \multicolumn{3}{c}{ Rapazes $(\mathbf{n = 4 5})$} & \multicolumn{3}{c}{ Moças $(\mathbf{n}=\mathbf{3 3})$} \\
\cline { 2 - 7 } & Baixa ACR & Alta ACR & Valor de $\mathbf{p}$ & Baixa ACR & Alta ACR & Valor de $\boldsymbol{p}$ \\
\hline ACR $(\%)^{\dagger \dagger}$ & $57,80 \%$ & $42,20 \%$ & $0,297^{*}$ & $39,40 \%$ & $60,60 \%$ & $0,223^{*}$ \\
Idade (anos) & $15,1(0,4)$ & $15,1(0,3)$ & 0,845 & $15,2(0,4)$ & $15,1(0,3)$ & 0,235 \\
Massa corporal $(\mathrm{kg})$ & $64,8(16,0)$ & $54,8(11,3)$ & 0,006 & $55,3(15,2)$ & $50,6(10,0)$ & 0,087 \\
Estatura $(\mathrm{cm})$ & $171,6(6,5)$ & $172,1(3,7)$ & 0,646 & $161,8(10,0)$ & $160,0(9,1)$ & 0,501 \\
IMC $\left(\mathrm{kg} / \mathrm{m}^{2}\right)$ & $22,1(4,7)$ & $18,9(3,3)$ & 0,004 & $20,3(4,6)$ & $19,5(2,6)$ & 0,137 \\
VO $_{2}$ máx $(\mathrm{mL} / \mathrm{kg} / \mathrm{min})$ & $40,6(5,1)$ & $46,1(2,6)$ & 0,001 & $31,6(0,8)$ & $35,4(3,0)$ & 0,001 \\
\hline
\end{tabular}

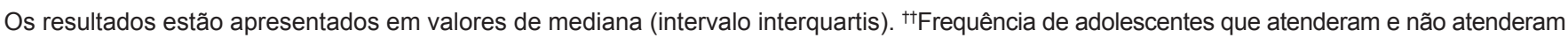
ao critério do Fitnessgram para aptidão cardiorrespiratória; *Teste do qui-quadrado. 
Tabela 3 - Indicadores de adiposidade corporal de adolescentes segundo aptidão cardiorrespiratória (ACR) e sexo

\begin{tabular}{llcclcc}
\hline & \multicolumn{3}{c}{ Rapazes $(\mathbf{n}=\mathbf{4 5})$} & \multicolumn{3}{c}{ Moças $(\mathbf{n}=\mathbf{3 3})$} \\
\cline { 2 - 7 } & Baixa ACR & Alta ACR & Valor de $\boldsymbol{p}$ & Baixa ACR & Alta ACR & Valor de $\boldsymbol{p}$ \\
\hline DCTR $(\mathrm{mm})$ & $15,5(9,6)$ & $9,1(6,2)$ & 0,001 & $22,8(7,9)$ & $17,9(8,3)$ & 0,009 \\
$\operatorname{DCSE}(\mathrm{mm})$ & $14,4(13,2)$ & $9,2(4,5)$ & 0,003 & $21,2(15,9)$ & $14,2(7,0)$ & 0,033 \\
LDC $(\mathrm{mm})$ & $32,6(19,0)$ & $18,7(9,6)$ & 0,001 & $43,5(24,8)$ & $32,0(13,2)$ & 0,008 \\
\%GORD & $25,6(11,7)$ & $15,6(8,7)$ & 0,001 & $31,8(6,0)$ & $27,5(7,4)$ & 0,011 \\
\hline
\end{tabular}

Os resultados estão apresentados em valores de mediana (intervalo interquartis). DCTR: dobra cutânea tricipital; DCSE: dobra cutânea subescapular; $\Sigma \mathrm{DC}$ : somatória das dobras cutâneas tricipital e subescapular; \%GORD: gordura corporal relativa.

Tabela 4 - Correlação entre aptidão cardiorrespiratória (número de voltas completadas no SR-20m), consumo máximo de oxigênio (Vo máx) e indicadores de adiposidade corporal em adolescentes de acordo com sexo

\begin{tabular}{llllll}
\hline & IMC & DCTR & DCSE & IDC & \%GORD \\
\hline Rapazes & & & & & \\
SR-20m & $-0,360^{*}$ & $-0,488^{* *}$ & $-0,441^{* *}$ & $-0,475^{* *}$ & $-0,441^{* *}$ \\
VO ${ }_{2}$ máx & $-0,378^{*}$ & $-0,507^{* *}$ & $-0,500^{* *}$ & $-0,513^{* *}$ & $-0,480^{* *}$ \\
Moças & & & & \\
SR-20m & $-0,203$ & $-0,444^{* *}$ & $-0,418^{*}$ & $-0,481^{* *}$ & $-0,472^{* *}$ \\
VO $_{2}$ máx & $-0,101$ & $-0,363^{*}$ & $-0,354^{*}$ & $-0,404^{*}$ & $-0,396^{*}$ \\
\hline
\end{tabular}

IMC: índice de massa corpórea; DCTR: dobra cutânea tricipital; DCSE: dobra cutânea subescapular; $\Sigma$ DC: somatória das dobras cutâneas tricipital e subescapular; \%GORD: gordura corporal relativa. ${ }^{* *} p<0,01$ e ${ }^{*} 0,01<p<0,05$.

ACR, tanto para os rapazes quanto para as moças, embora se observem diferenças significativas na proporção de baixa ACR entre rapazes $(57,8 \%)$ e moças $(39,4 \%)(p=0,037)$.

Houve diferença na massa corporal e IMC para o sexo masculino, com valores mais elevados nos adolescentes que não atenderam ao critério para ACR. Vale destacar que os rapazes com ACR satisfatória apresentaram menores valores de massa corporal $(15,5 \%)$ e de IMC (14,5\%), em comparação aos seus pares com baixa ACR. Para o $\mathrm{VO}_{2}$ max, as diferenças entre ACR satisfatória e não-satisfatória foram de 13 e $12 \%$ para rapazes e moças, respectivamente.

Com relação aos indicadores de adiposidade corporal utilizados, tanto os rapazes quanto as moças com alta ACR apresentaram sistematicamente valores mais baixos de adiposidade do que seus pares com baixa ACR, embora em diferentes magnitudes (Tabela 3).

Para os rapazes, os valores da DCSE foram $36 \%$ menores para aqueles que atenderam ao critério da ACR em relação aos que não atenderam. Resultados semelhantes foram encontrados na DCTR (38\%), \%GORD (39\%) e SDC (42\%). Esse fato também pode ser verificado entre as moças, embora as diferenças entre as adolescentes com alta e baixa ACR tivesssem magnitude menor $(\% \mathrm{GORD}=14 \%$; $\mathrm{DCTR}=21 \%$; $\mathrm{SDC}=26 \%$; $\mathrm{DCSE}=33 \%$ ).

Na Tabela 4, são apresentados os valores de correlação entre indicadores da ACR (número de voltas no teste SR-20m e $\mathrm{VO}_{2}$ max) e de adiposidade corporal. Verificou-se, entre os rapazes, correlações negativas moderadas e estatisticamente significativas tanto para o teste SR-20m quanto para o $\mathrm{VO}_{2}$ máx, variando de $-0,441$ a $-0,513$, exceto para o IMC, que apresentou baixa correlação, tanto nos rapazes quanto nas moças. Nas moças, de forma semelhante aos rapazes, os demais indicadores de adiposidade corporal se correlacionaram moderadamente, de forma inversamente proporcional e significativa.

\section{Discussão}

Muitos estudos indicam que o excesso de gordura corporal na infância e na adolescência pode trazer inúmeras complicações para a saúde, uma vez que esse fato está associado a desordens psicológicas e sociais, problemas ortopédicos e disfunções metabólicas, tais como dislipidemias, hipertensão arterial, intolerância à glicose e aumento 
da resistência periférica à insulina ${ }^{(27,28)}$. Por outro lado, uma boa ACR em crianças e adolescentes pode contribuir para a redução de fatores de risco cardiovascular ${ }^{(9-11)}$, bem como pode auxiliar o controle dos depósitos de gordura corporal $^{(17,18)}$. Portanto, investigar a relação entre esses fatores nessa faixa etária pode trazer valiosas contribuições para prevenir sobrepeso/obesidade e promover a saúde cardiovascular.

Os resultados do presente estudo, de forma semelhante ao encontrado em adolescentes de Florianópolis ${ }^{(29)}$, indicaram uma elevada prevalência de adolescentes com baixa ACR, tanto no sexo masculino (60\%) quanto no feminino (40\%). Esses achados confirmam informações anteriormente produzidas em crianças de sete a dez anos de idade da mesma escola investigada que mostraram prevalência de baixa ACR em $32 \%$ das meninas e $27 \%$ dos meninos ${ }^{(30)}$, sugerindo que a ACR tende a ser ainda mais comprometida com a passagem da infância para a adolescência.

Embora esta investigação não tenha analisado o comportamento de parâmetros metabólicos importantes para a saúde, os resultados encontrados são preocupantes, uma vez que estudos epidemiológicos têm demonstrado forte associação entre baixos índices de ACR e fatores de risco cardiovascular e síndrome metabólica em populações jovens ${ }^{(9-11)}$.

Neste estudo, os maiores valores de adiposidade corporal foram encontrados nos adolescentes com baixa ACR, em ambos os sexos. Tal fato pode ter contribuído, pelo menos em parte, para a elevada prevalência de baixa ACR observada, uma vez que indivíduos com maior massa corporal e, sobretudo, com quantidade de gordura corporal mais elevada, tendem a apresentar dificuldades de locomoção, diminuição na frequência de passadas e menor estabilidade durante a caminhada e/ou corrida ${ }^{(31)}$.

Com base nos indicadores de adiposidade corporal observados neste estudo, verificou-se que índices satisfatórios da ACR parecem contribuir de alguma maneira para o controle dos depósitos de gordura corporal em adolescentes de ambos os sexos. Nesse sentido, os valores dos indicadores da gordura corporal total, de membros e de tronco foram aproximadamente $30 \%$ menores para os adolescentes com alta ACR em relação aos seus pares com baixa ACR. Entretanto, a confirmação ou não dessa hipótese é dependente de estudos com delineamento longitudinal que controlem outras variáveis importantes, tais como os hábitos alimentares e o nível de atividade física habitual.
Klasson-Heggeb $\varnothing$ et a ${ }^{(17)}$ relataram uma forte associação entre o aumento da ACR e a redução dos valores da somatória de dobras cutâneas em crianças de nove anos e adolescentes de 15 anos de ambos os sexos. Adicionalmente, índices elevados de ACR parecem favorecer a redução da adiposidade corporal em crianças ${ }^{(18)}$, indicando que uma boa ACR pode exercer papel importante na prevenção e controle do excesso de gordura corporal.

Nesse sentido, uma meta-análise envolvendo 14 estudos publicados até 2004, com 481 meninos e meninas com sobrepeso de aproximadamente 12 anos, indicou que 155 180 minutos por semana de prática de exercícios aeróbios de moderada a alta intensidade reduzem a adiposidade corporal $^{(32)}$. Na mesma direção, Watts et al ${ }^{(33)}$ afirmam, com base em uma revisão sistemática, que a prática regular de exercícios aeróbios pode contribuir tanto para a redução da gordura corporal quanto para o aumento da massa magra, além de favorecer o aumento da ACR e da força muscular em crianças e adolescentes obesos.

As correlações encontradas no presente estudo entre indicadores de ACR ( ${ }^{\circ}$ de voltas e $\mathrm{VO}_{2}$ máx) e de adiposidade corporal indicam que a melhoria do desempenho no teste SR-20m (aumento no número de voltas e no $\mathrm{VO}_{2}$ máx) parece estar associada, pelo menos em parte, a reduzidos depósitos de gordura corporal em adolescentes de ambos os sexos. Por outro lado, acredita-se que a elevação da adiposidade corporal possa influenciar negativamente a ACR. Entretanto, apesar de as correlações terem sido moderadas e negativas, em virtude do delineamento adotado nesta investigação (estudo transversal), não foi possível estabelecer a relação de causa e efeito.

A redução do desempenho no teste SR-20m pode ser explicada por uma queda na ACR, pelo aumento dos depósitos de gordura corporal ou, ainda, por ambos. Pesquisadores que estudaram esse fenômeno em crianças e adolescentes, com base em informações produzidas em 11 países de 1980 a 2000, identificaram uma tendência de queda no desempenho na ordem de $0,43 \%$ com o avanço da idade ${ }^{(34)}$. Embora o declínio da aptidão aeróbia possa estar relacionado ao aumento da gordura corporal, tais pesquisadores defendem que a maior causa da queda de performance aeróbia em adolescentes está ligada à diminuição da atividade física habitual.

Apesar do estudo apresentar algumas limitações, como a adoção de um delineamento transversal, a ausência de informações sobre atividade física habitual, hábitos alimentares e comportamento metabólico dos sujeitos investigados, os 
resultados encontrados são preocupantes, visto que grande parte dos adolescentes apresentou baixa ACR. Além disso, esse fato associado a valores elevados de adiposidade corporal pode aumentar a chance do desenvolvimento precoce dos fatores de risco cardiovascular e de síndrome metabólica nessa população $0^{(10,35)}$.

Assim, pode-se concluir que os adolescentes de ambos os sexos com alta ACR tendem a apresentar menores valores de adiposidade corporal. A correlação encontrada neste estudo entre menor ACR e maior adiposidade corporal sugere que o risco cardiovascular pode aumentar sensivelmente em indivíduos com sobrepeso/obesidade. Sugere-se, ainda, que estudos com delineamento longitudinal e que incluam a avaliação de indicadores metabólicos, bem como o controle de hábitos alimentares e da atividade física habitual, possam ampliar as análises das informações produzidas nesta investigação.

\section{Agradecimentos}

Os autores agradecem as bolsas concedidas pelo Conselho Nacional de Desenvolvimento Científico e Tecnológico (CNPq) e pela Coordenação de Aperfeiçoamento de Pessoal de Nível Superior (Capes).

\section{Referências bibliográficas}

1. Hancox RJ, Poulton R. Watching television is associated with childhood obesity: but is it clinically important? Int J Obes (Lond) 2006;30:171-5.

2. Janz KF, Levy SM, Burns TL, Torner JC, Willing MC, Warren JJ. Fatness, physical activity, and television viewing in children during the adiposity rebound period: the lowa Bone Development Study. Prev Med 2002;35:563-71.

3. Ribeiro RQ, Lotufo PA, Lamounier JA, Oliveira RG, Soares JF, Botter DA. Additional cardiovascular risk factors associated with excess weight in children and adolescents: the Belo Horizonte heart study. Arq Bras Cardiol 2006;86:408-18.

4. Deshmukh-Taskar P, Nicklas TA, Morales M, Yang SJ, Zakeri I, Berenson GS. Tracking of overweight status from childhood to young adulthood: the Bogalusa Heart Study. Eur J Clin Nutr 2006;60:48-57.

5. Lohman TG, Ring K, Pfeiffer K, Camhi S, Arredondo E, Pratt C et al. Relationships among fitness, body composition, and physical activity. Med Sci Sports Exerc 2008;40:1163-70.

6. Rowlands AV, Eston RG, Ingledew DK. Relationship between activity levels, aerobic fitness, and body fat in 8- to 10-yr-old children. J Appl Physiol 1999;86:1428-35.

7. LaMonte MJ, Barlow CE, Jurca R, Kampert JB, Church TS, Blair SN. Cardiorespiratory fitness is inversely associated with the incidence of metabolic syndrome: a prospective study of men and women. Circulation 2005;112:505-12.

8. Blair SN, Cheng Y, Holder JS. Is physical activity or physical fitness more important in defining health benefits? Med Sci Sports Exerc 2001;33:S379-99.

9. Ruiz JR, Ortega FB, Rizzo NS, Villa I, Hurtig-Wennlof A, Oja L et al. High cardiovascular fitness is associated with low metabolic risk score in children: the European Youth Heart Study. Pediatr Res 2007;61:350-5.

10. Eisenmann JC, Welk GJ, Ihmels M, Dollman J. Fatness, fitness, and cardiovascular disease risk factors in children and adolescents. Med Sci Sports Exerc 2007;39:1251-6.

11. Anderssen SA, Cooper AR, Riddoch C, Sardinha LB, Harro M, Brage S et al. Low cardiorespiratory fitness is a strong predictor for clustering of cardiovascular disease risk factors in children independent of country, age and sex. Eur J Cardiovasc Prev Rehabil 2007;14:526-31.

12. Carnethon MR, Gulati M, Greenland P. Prevalence and cardiovascular disease correlates of low cardiorespiratory fitness in adolescents and adults. JAMA 2005;294:2981-8.

13. Andersen LB, Hasselstrom H, Gronfeldt V, Hansen SE, Karsten F. The relationship between physical fitness and clustered risk, and tracking of clustered risk from adolescence to young adulthood: eight years follow-up in the Danish Youth and Sport Study. Int J Behav Nutr Phys Act 2004;1:6.
14. Shaibi GQ, Cruz ML, Ball GD, Weigensberg MJ, Kobaissi HA, Salem GJ et al. Cardiovascular fitness and the metabolic syndrome in overweight latino youths. Med Sci Sports Exerc 2005;37:922-8.

15. Eisenmann JC, Wickel EE, Welk GJ, Blair SN. Relationship between adolescent fitness and fatness and cardiovascular disease risk factors in adulthood: the Aerobics Center Longitudinal Study (ACLS). Am Heart J 2005;149:46-53.

16. Twisk JW, Kemper HC, Van Mechelen W. Prediction of cardiovascular disease risk factors later in life by physical activity and physical fitness in youth: general comments and conclusions. Int J Sports Med 2002;23 (Suppl 1): S44-9.

17. Klasson-Heggebø L, Andersen LB, Wennlof AH, Sardinha LB, Harro M, Froberg $\mathrm{K}$ et al. Graded associations between cardiorespiratory fitness, fatness, and blood pressure in children and adolescents. Br J Sports Med 2006;40:25-9.

18. Nassis GP, Psarra G, Sidossis LS. Central and total adiposity are lower in overweight and obese children with high cardiorespiratory fitness. Eur J Clin Nutr 2005;59:137-41.

19. Ross WD, Marfell-Jones MJ. Kinanthropometry. In: MacDougall JD, Wenger HA, Green HS, editors. Physiological testing of the elite athlete. Ithaca: Movement Publications; 1982. p. 75-115.

20. Gordon CC, Chumlea WC, Roche AF. Stature, recumbent length, and weight. In: Lohman TG, Roche AF, Martorell R, editors. Anthropometric standardization reference manual. Champaign: Human Kinetics Books; 1988. p. 3-8.

21. Harrison GG, Buskirk ER, Carter LJE, Johnston FE, Lohman TG, Pollock ML et al. Skinfold thicknesses and measurement technique. In: Lohman TG, Roche AF, Martorell R, editors. Anthropometric standardization reference manual. Champaign: Human Kinetics Books; 1988. p. 55-70.

22. Boileau RA, Lohman TG, Slaughter MH. Exercise and body composition in children and youth. Scand J Sports Sci 1985;17:17-27.

23. Leger L, Lambert J, Goulet A, Rowan C, Dinelle Y. Aerobic capacity of 6 to 17-year-old Quebecois--20 meter shuttle run test with 1 minute stages. Can J Appl Sport Sci 1984;9:64-9.

24. Leger LA, Mercier D, Gadoury C, Lambert J. The multistage 20 meter shuttle run test for aerobic fitness. J Sports Sci 1988;6:93-101.

25. Cooper Institute for Aerobics Research. The prudential FITNESSGRAM test administration manual. Champaign: Human Kinetics Books; 1999.

26. Suminski RR, Ryan ND, Poston CS, Jackson AS. Measuring aerobic fitness of Hispanic youth 10 to 12 years of age. Int J Sports Med 2004;25:61-7.

27. Zhang CX, Tse LA, Deng XQ, Jiang ZQ. Cardiovascular risk factors in overweight and obese Chinese children: a comparison of weight-for-height index and BMI as the screening criterion. Eur J Nutr 2008;47:244-50.

28. Katz DL, O'Connell M, Yeh MC, Nawaz H, Njike V, Anderson LM et al. Public health strategies for preventing and controlling overweight and obesity in 
school and worksite settings: a report on recommendations of the Task Force on Community Preventive Services. MMWR Morb Mortal Wkly Rep 2005;54:1-12.

29. Vasques DG, Silva KS, Lopes AS. Aptidão cardiorrespiratória de adolescentes de Florianópolis, SC. Rev Bras Med Esporte 2007;13:376-80.

30. Ronque ERV, Cyrino ES, Dórea VR, Serassuelo Júnior H, Galdi EHG, Arruda M. Diagnóstico da aptidão física em escolares de alto nível socioeconômico: avaliação referenciada por critérios de saúde. Rev Bras Med Esporte 2007;13:71-6.

31. Wearing SC, Hennig EM, Byrne NM, Steele JR, Hills AP. The impact of childhood obesity on musculoskeletal form. Obes Rev 2006;7:209-18.
32. Atlantis E, Barnes EH, Singh MA. Efficacy of exercise for treating overweight in children and adolescents: a systematic review. Int J Obes (Lond) 2006;30:1027-40.

33. Watts K, Jones TW, Davis EA, Green D. Exercise training in obese children and adolescents: current concepts. Sports Med 2005;35:375-92.

34. Tomkinson GR, Leger LA, Olds TS, Cazorla G. Secular trends in the performance of children and adolescents (1980-2000): an analysis of 55 studies of the 20m shuttle run test in 11 countries. Sports Med 2003;33:285-300.

35. Sakuragi S, Abhayaratna K, Gravenmaker KJ, O'Reilly C, Srikusalanukul W, Budge MM et al. Influence of adiposity and physical activity on arterial stiffness in healthy children: The Lifestyle of Our Kids Study. Hypertension 2009;53:611-6. 